\title{
The size estimation of injection drug users (IDUs) using the network scale-up method (NSUM) in Iranshahr, Iran
}

\author{
Sakineh Narouee ${ }^{1}$, Mohsen Shati ${ }^{2}$, Mahshid Nasehi*3, Farhad Dadgar ${ }^{4}$
}

Received: 1 Nov 2018

Published: 30 Dec 2019

\begin{abstract}
Background: The size estimation of key populations is a necessary part of surveillance systems to access global human immunodeficiency virus (HIV) infection. In this study, the NSU method was used to estimate injection drug users (IDUs).

Methods: NSU method was performed on 1000 individuals in Iranshahr province with street-based sampling from 2016 to 2017 . A questionnaire comprising items on demographic information and items measuring the network size of participants about IDUs was administered. The estimated size was adjusted for transmission error and barrier effect with PF and VF factors. The relationship between knowing IDUs and demographic variables was assessed using logistic regression. All analyses were performed in SPSS 19 and Microsoft Excel.

Results: In this study, 500 men and 500 women were included. The average age (standard deviation) of the participants was 29.6 (7.8) years. IDUs were estimated at 1263 per 100000 population of Iranshahr in Sistan and Baluchestan province, Iran. The estimated size was 7.5 times more in men than in women (2766 vs 364). Moreover, the highest estimated size belonged to the population of 18 30 -year-olds (1187). Sex and education level had significant relationships with knowing IDUs ( $<0.001)$.

Conclusion: To improve preventive programs, the number of outreach teams must be increased to have access to IDUs, educate them, and upgrade the coverage of harm-reduction services. According to cultural considerations and the illegal nature of injection drug use, social mobilization is essential to reduce the stigma.
\end{abstract}

Keywords: Network scale-up, Size estimation, Injection drug users, Iran

Conflicts of Interest: None declared

Funding: Iran University of Medical Sciences

*This work has been published under CC BY-NC-SA 1.0 license.

Copyright $₫$ Iran University of Medical Sciences

Cite this article as: Narouee S, Shati M, Nasehi M, Dadgar F. The size estimation of injection drug users (IDUs) using the network scale-up method (NSUM) in Iranshahr, Iran. Med J Islam Repub Iran. 2019 (30 Dec);33:158. https://doi.org/10.47176/mjiri.33.158

\section{Introduction}

In many countries, the human immunodeficiency virus (HIV) epidemic is concentrated in subgroups under the key population with the capacity of transmitting HIV to the general population, including female sex workers (FSWs), injection drugs users (IDUs), and men who have sex with men (MSMs) $(1,2)$.

Population size estimations (PSE) for key populations at national and local levels are essential components for revising strategic planning, appropriately allocating resources, and advocating for services for those populations

Corresponding author: Dr Mahshid Nasehi, nasehi.m@iums.ac.ir

1. Department of Health, Iranshahr University of Medical Sciences, Sistan and Baluchestan, Iran

2. Mental Health Research Center, School of Behavioral Sciences and Mental Health, Tehran Institute of Psychiatry, Iran University of Medical Sciences, Tehran, Iran

3. Department of Epidemiology, School of Public Health, Iran University of Medical Sciences, Tehran, Iran

4. Iranshahr University of Medical Sciences, Sistan and Baluchestan, Iran
$(3,4)$. The collection of this information is difficult and may be prone to bias using traditional and direct sampling methods due to the stigmatized and illegal nature of this behavior, which limits the participation of the key population in a survey (3-5). Indirect methods have recently been introduced to estimate key populations, including the network scale-up method (NSUM) $(3,5)$. In addition to other indirect methods such as enumeration, capture-recapture, and multiplier in which access to reliable data sources is limited, the low cost and simplicity of

$\uparrow$ What is "already known" in this topic:

Network scale-up (NSU) with an indirect approach is one of the best data collection methods about hidden populations.

\section{$\rightarrow$ What this article adds:}

Assessment of the community to understand its health status, concerns, and the provision of health services is done through knowledge, data collection, analysis, and dissemination on the characteristics, strengths, resources, and needs of the community, with the help of community members. 
the NSUM make it an appropriate method for size estimation. The indirect nature of data collection in this method provides a concurrent estimation of different subpopulations. The average number of key populations reported by every respondent constitutes a fraction of their active social networks. This fraction scales up a fraction of the total population, and thus yields an estimate of the number of key populations of interest $(3,6-8)$.

The Islamic Republic of Iran is a major transit country for illicit drugs, especially because of its proximity to Pakistan and its border with Afghanistan, which is the main producer of opium in the world (9). In the Islamic Republic of Iran in 2016, 66000 people were living with HIV infection, of whom 5000 were newly infected (10). The prevalence of HIV infection has been established to be higher among IDUs compared to the general population as an important HIV mode of transmission in Iran (11). The $\mathrm{HIV}$ epidemic is concentrated in subgroups of the population whose behavior exposes them to a high risk of acquiring HIV infection, including IDUs, FSWs, and MSMs (1). A study in Iran showed that the prevalence of IDUs was 280 per 100000 populations.

Sistan and Baluchestan province is located in Southeast Iran, bordering with Pakistan and Afghanistan. The city of Iranshahr is located in the center of this province, close to the city of Sarbaz, on the path of foreigners and drug transit. The present study was conducted in 2016-2017 to estimate the IDUs in Iranshahr, with the population of 265 316 in 2015. It is vital for HIV control program authorities at the national and local levels to have access to more relevant information on the number of key groups that increase the risk of HIV or they are at high risk of HIV. By doing so, they can offer proper educational programs and increase harm reduction services to decrease HIV incidence and transmission in this key group.

\section{Methods}

In this cross sectional study, 1000 individuals were recruited from all over Iranshahr. Of them, 71\%, 25\%, and $4 \%$ were surveyed in central, Bampur, and Bazman districts, respectively, and equally composed of men and women. The survey was conducted with a nearly equal sampling ratio of 49.8 in urban and 50.2 in rural areas. The sample was selected using the street-based simple sampling method from among those who were alone in crowded areas such as streets, parks, hospitals, clinics, and taxi stations. Interested persons were those aged over 18 years who had lived in Iranshahr for at least 5 years. The questionnaire contained 2 sections: The first part, demographic information, included items on sex, age, occupation, level of education, place of residence, and marital status; and the second part comprised questions related to IDUs. The research objectives were explained to the participants and informed consent was obtained in a face-toface manner. Then, the questionnaire was completed by 4 local trained reviewers, respecting sex-matching between the reviewer and the participant.

A basic formula for calculating personal network size is $\mathrm{e} / \mathrm{t}=\mathrm{m} / \mathrm{c}$. Here, e is the size of the reference subpopulation, $\mathrm{t}$ the size of the total population, $\mathrm{m}$ the average num- ber of people belonging to a reference subpopulation who were known by our sample, and c the active network size. Combining the information of several reference groups, the above formula can be modified to obtain better estimates: $\hat{c}_{i}=\left(\sum_{j} m_{i j} / \sum_{j} e_{j}\right)$.t.

In this study, the definition of "knowing" was as follows: "people whom you know and who know you by sight or by name; with whom you can interact, if needed, and with whom you have contacted over the last 2 years in person, over the phone, or by email" (6). The respondents were asked how many people they knew who had injected drugs at least once in the previous year; then, they were stratified by gender and placed into 3 age groups $(<18,18$ $30,>30$ years).

Usually, participants count a large number of people in target groups. Thus, in many studies to increase the accuracy of key population estimation, the data are cleaned extensively before data analysis. Therefore, in this study, responses above 30 were rounded to 30 (12). The estimation error was corrected by eliminating cases belonging to high-risk groups whom the participants knew but were not sure about their high-risk behavior. The aim of this correction was to reach the minimum size of the population of high-risk groups to resolve the risk of recall bias. The second correction was the removal of cases of high-risk groups who were known by the participants but resided outside Iranshahr. One important assumption in the NSUM is that the prevalence of drug use in the entire population can be estimated by calculating the proportion of IDUs in the social network of a representative sample of the general population $(\mathrm{m} / \mathrm{c})$. Thus, the size of the population of IDUs was estimated based on the formula $\hat{\mathrm{e}}_{\text {crude }}=t \cdot \frac{\sum m_{i j}}{\sum c_{i}}$. In this formula, ê estimates the population size of the target group (IDUs), $\mathrm{m}_{\mathrm{ij}}$ the number of individuals who $\mathrm{i}$ knows from the target group $\mathrm{j}, \mathrm{t}$ denotes the total number of base population in Iranshahr, and cij is the size of the social network, which is considered to be 308.35 as an important component in the NSUM. The average number of IDUs in the personal network of participants $(\mathrm{m})$ was calculated by asking them how many IDUs they knew.

The NSU method assumes that respondents are aware of the behavior of other members of their network and that the members of the general population have an equal chance of knowing anyone in the target group. Thus, in this study, the estimates were adjusted based on 2 correction factors: barrier and transmission ratios. The barrier effect is an error made by geographic, social, or other factors because of which respondents know more or less than the expected number of IDUs. Moreover, in transmission error, the possibility of transferring information about individual behaviors to social network members is not likely to be equal. This effect makes people less likely to report IDUs in their network because of community stigma or inadequate information. Since the NSUM assumes that respondents are aware of the behavior of other members of their network and that the members of the general population have an equal chance of knowing anyone in the target group, popularity factor (PF) and visibility factor 
(VF) were also taken into account. PF is the ratio of the average social network size of the IDUs to the network size of the general population. The barrier effect can be identified and resolved under PF. Also, to eliminate the transmission error in the estimation of IDUs, VF was used. This is the percentage of social network members who are aware of the behavior of high-risk individuals. Accordingly, the barrier ratio and transmission ratio for IDUs equaled 0.7 and 0.54 , respectively (In other words, it was assumed that only around half of the social network of each person in Iranshahr had knowledge about the injection drug consumption behaviors.). The crude NSU estimate was divided by VF and PF to adjust for these 2 sources of bias. The equation $\hat{\mathrm{e}}_{\text {crude }} * \frac{1}{\text { popularity factor }} *$ $\frac{1}{\text { visibility factor }}$ was used to estimate the $95 \%$ confidence interval for the estimation of IDUs $(13,14)$. Thus, to investigate the relationship between knowing IDUs and demographic variables such as age, sex, marital status, education level, occupation, and residence, a univariate analysis was first conducted. The variables, whose $\mathrm{p}$ value in this part of the analysis was $<0.2$, entered the logistic regression model. All analyses were performed in SPSS 19 and Microsoft Excel.

\section{Results}

In total, 500 men and 500 women were studied. The analysis of data belonging to 1000 participants from 3
Table 1. Demographic information of the participants

\begin{tabular}{llc}
\hline Variable & & $\begin{array}{c}\text { Frequency } \\
(\%)\end{array}$ \\
\hline Sex & Male & $500(50)$ \\
Age (years) & Female & $500(50)$ \\
& $25-24$ & $293(29.3)$ \\
& $59-49$ & $597(59.7)$ \\
Marital status & Single & $108(10.8)$ \\
& Married & $2(0.2)$ \\
Level of & Divorced/widowed & $250(25.0)$ \\
education & Illiterate/elementary school & $721(72.1)$ \\
& Junior high school & $29(2.9)$ \\
& High school diploma & $198(19.8)$ \\
& Graduate degree & $226(22.6)$ \\
Occupation & Postgraduate degree & $404(40.4)$ \\
& Employee & $167(16.7)$ \\
& Service provider & $5(0.5)$ \\
& University student & $73(7.3)$ \\
& Homemaker & $398(39.8)$ \\
Place of & Unemployed/in military service & $93(9.3)$ \\
residence & Urban & $369(3.69)$ \\
\hline & Rural & $67(6.7)$ \\
& & $498(49.8)$ \\
& & $502(50.2)$ \\
\hline
\end{tabular}

districts of Iranshahr revealed that participants' age ranged from 18 to 85 years, and $59.7 \%$ of them were $25-39$ years old. The average age (standard deviation) was 29.6 (7.8) years, $29.4(0.3)$ for men and 29.9(0.3) for women. Over half of the participants were married $(72.1 \%)$ and had a high school diploma (40.4\%) (Table 1).

In addition, $89.34 \%$ of IDUs were reported to be addict-

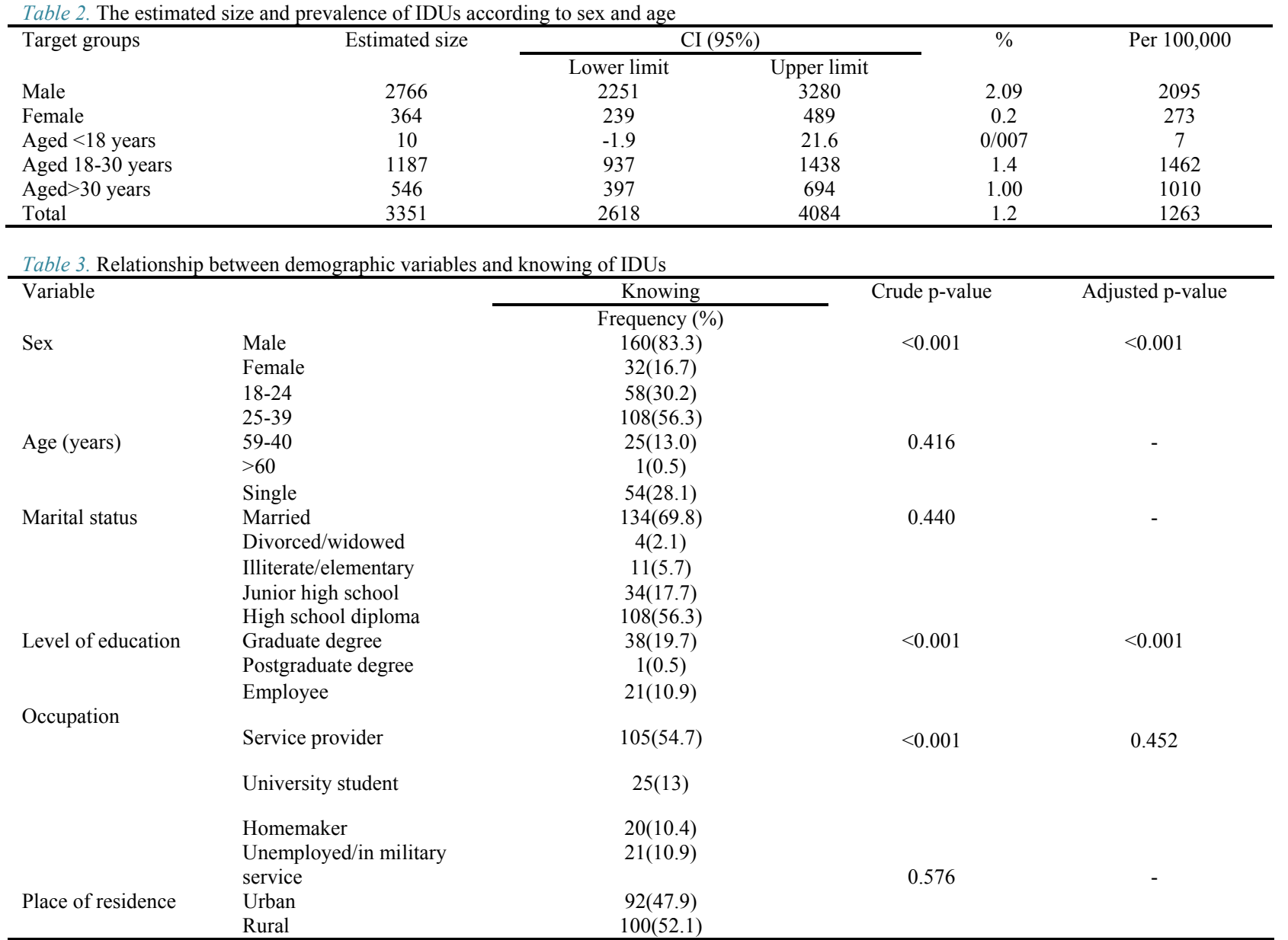


ed. Participants knew $0.3 \%, 40.2 \%$, and $59.5 \%$ of IDUs who had injected at least once in the past year and aged $<18,18-30$, and over 30 years, respectively. Moreover, $93.67 \%$ (1126 people) reported that the users were male.

In the present study, $19.2 \%$ of the participants knew at least one injectable user. Results indicated that the crude estimation of IDUs was 512.2 per 100000 persons. To adjust common errors in NSUM with the used VF and PF, the estimation of IDUs changed to 1263 per 100000 persons. Table 2 presents the total estimated size and prevalence of IDUs per 100 and 100000 persons. The difference between men and women was related to injected drugs in which the estimated size for men was 7.5 times more than for women (2766 vs 364). Furthermore, Table 2 presents the estimated size of IDUs in Iranshahr based on sex and age. From among the 3 age groups, the highest estimated size belonged to the population aged 18-30 years (1187).

Based on Table 3, male participants, those aged 25 to 39 years, married people, university graduates, service providers, and people living in the town knew more IDUs. This table demonstrates the frequency of knowing IDUs by demographic variables. In the univariate analysis of sex, education level, and occupation, there was a significant statistical relationship with knowing IDUs $(\mathrm{p}<0.001)$. However, in the multivariate analysis, the probability of knowing IDUs is lower in women (odds ratio: 0.7 ) than in men, and it is more in individuals with higher education level (odds ratio: 1.5) than others, demonstrating a significant difference $(\mathrm{p}<0.001)$.

\section{Discussion}

The estimation of IDUs in Iranshahr is required for local HIV control efforts. The findings of this study showed that the estimated size of the population with injection drugs (PWID) users was 3351, corresponding to $1.2 \%$ in the general population of Iranshahr. In a study in Iran, the number of IDUs was estimated at 208000 . According to the statistics reported by Iranian Ministry of Health and Medical Education, the estimated size of illicit drug users is around $200000(6,13)$. Based on a pilot study in Kerman, the lowest prevalence of a specific drug use was $1.2 \%$, which corresponded to injecting drugs. Also, the average sample size in each province was approximately 400 , ranging from 200 to $1000(6,15)$. Similar to other population size estimation, NSU is prone to a number of biases, including imperfect knowledge of the behaviors of the person in his/her social network and the influences of stigma on the visibility of certain behaviors and overestimation due to differences in understanding the concept of IDUs with what is being studied (16). The difference between urban and rural areas and higher sample sizes may be due to differences in the results of the study. The size estimated in the present study was consistent with results reported by a study employing the probability method in Kerman, but was less than the values reported for Tabriz and those reported using the frequency method for Kerman $(15,16)$. Moreover, estimating the number of IDUs in the United States through a meta-analysis showed that lifetime PWID equaled $2.6 \%$. The population estimate of the past year was $0.30 \%$ or 774434 PWID (17). In a study in Ukraine, after the removal of the effect of response bias, the number of IDUs was estimated at 358000 .

NSUM builds upon the assumption that everyone in the general public has an equal chance of knowing members of a group and everyone has perfect knowledge about his/her acquaintances. Nevertheless, these assumptions rarely hold due to stigma and discrimination and, therefore, results are generally biased. Furthermore, it is difficult to obtain reliable population size estimates due to the widespread stigma and discrimination against these populations. The results of the NSU method may be underestimated because of the response bias (eg, Respondents may fail or be reluctant to accurately report themselves or their acquaintances.) and information transmission bias (For instance, respondent may not be aware of such behaviors among their acquaintances in the network.). The severity of stigma to substance abuse and drug use patterns for cultural, social, and religious reasons can lead to the underestimation of the results $(15,18)$

In this study, less than $20 \%$ of people knew at least one IDU. This value was $3.9 \%$ in a study in China (7). The fact that $70 \%-80 \%$ of the community do not know about IDUs and do not know even one person belonging to this group may be due to the fact only a very small group of people are associated with IDUs or that these people do not know the risky behavior of IDUs.

The findings of the present study revealed that the highest prevalence of IDUs belonged to the 18-30-year-olds in Iranshahr. Based on the results, the prevalence of IDUs in those aged 18-30 years was 1.9\%. A study in Iran reported that no substantial difference existed between age groups above 30 and $18-30$ years (6). Also, in the city of Larestan, the prevalence of drug abuse in 18-30 age group was $2.5 \%$. Finally, in Tabriz, $56.5 \%$ of male IDUs were over 30 years old and $43.6 \%$ were $18-30$ years old $(14,19)$.

Findings of the present study indicated that the prevalence of IDUs was 7.5 times more in men than in women. The estimated number of men was 12 times higher than in women in Iran (6). In the US, the estimated number for men and women was $0.36 \%$ vs $0.21 \%$, respectively (17). In addition, estimation of the prevalence of risky behaviors using NSUM in Larestan showed that the prevalence of drug users was significantly higher in men than in women (19). In terms of sex, male participants were more likely to know IDUs. This may be due to the higher degree of response bias caused by stigma in women's behaviors compared to men's or by a higher bias in recalling female behavior due to their more hidden networks and negative pressure for this behavior $(7,19,20)$. In this study, similar to the study on the entire country, there was a significant relationship between the level of education and the knowledge of this high-risk group (6).

The present study was subject to several limitations. The first limitation was the small proportion of participants reporting injection drug use. The second was the difficulty of accessing female participants. Third, the low level of participation of the elderly indicated that the street-based method may not necessarily provide the best possible representative sample. Forth, participants have 
had different interpretations of the definition of IDUs and acquaintances, which may have affected the accuracy of the results.

\section{Conclusion}

Optimizing the estimation of the number of IDUs is important for program planning and addressing health inequities. NSUM is an indirect method for estimating hard-toreach populations and may provide a higher prevalence of IDUs since these behaviors are underreported in direct methods. It seems that a comprehensive review and evaluation in the field of program implementation by counseling and drop-in centers (DIC) is required to prevent unhealthy behaviors, identify weaknesses and provide strategies to improve the program. Furthermore, more than $50 \%$ of the residents live in Iranshahr villages. To enhance preventive programs, the number of outreach teams must be increased to gain access to IDUs, educate them, and upgrade the coverage of harm-reduction services. Achieving AIDS control goals through stigma, discrimination, and social segregation as well as unjustified access to undesirable health care and outcomes is not possible. (20). Consequently, according to cultural considerations and the illegal nature of injection drug use, social mobilization is essential for reducing the stigma toward this behavior.

\section{Acknowledgments}

This article was derived from an M.S. thesis at Iran University of Medical Sciences (IUMS). The authors would like to thank the Faculty of Medical Sciences and Health Services of Iranshahr for contributing to this study.

\section{Conflict of Interests}

The authors declare that they have no competing interests.

\section{References}

1. Mutagoma M, Kayitesi C, Gwiza A, Ruton H, Koleros A, Gupta N, et al. Estimation of the size of the female sex worker population in Rwanda using three different methods. Int J STD AIDS. 2015 Oct;26(11):810-4

2. Yu D, Calleja JMG, Zhao J, Reddy A, Seguy N. Estimating the size of key populations at higher risk of HIV infection: a summary of experiences and lessons presented during a technical meeting on size estimation among key populations in Asian countries. West Pacific Surveill response J WPSAR. 2014;5(3):43-9.

3. Bernard HR, Hallett T, Iovita A, Johnsen EC, Lyerla R, McCarty C, et al. Counting hard-to-count populations: the network scale-up method for public health. Sex Transm Infect. 2010 Dec;86 Suppl $2: 11-5$

4. Ezoe S, Morooka T, Noda T, Sabin ML, Koike S. Population size estimation of men who have sex with men through the network scaleup method in Japan. PLoS One. 2012.

5. Kazemzadeh Y, Shokoohi M, Baneshi MR, Haghdoost AA. The Frequency of High-Risk Behaviors Among Iranian College Students Using Indirect Methods: Network Scale-Up and Crosswise Model. Int J high risk Behav Addict. 2016;5(3):e25130.

6. Nikfarjam A, Shokoohi M, Shahesmaeili A, Haghdoost AA, Baneshi MR, Haji-Maghsoudi S, et al. National population size estimation of illicit drug users through the network scale-up method in 2013 in Iran.

7. Guo W, Bao S, Lin W, Wu G, Zhang W, Hladik W, et al. Estimating the Size of HIV Key Affected Populations in Chongqing, China, Using the Network Scale-Up Method. PLoS One [Internet]. 2013 [cited 2018 Apr 11];8(8). Available from: http://journals.plos.org/plosone/article/ file?id=10.1371/journal pone $.0071796 \&$ type $=$ printable

8. Nikfarjam A, Shokoohi M, Shahesmaeili A, Haghdoost AA, Baneshi MR, Haji-Maghsoudi S, et al. National population size estimation of illicit drug users through the network scale-up method in 2013 in Iran. Int J Drug Policy. 2016;31:147-52.

9. United Nations Office on Drugs and Crime (UNODC). Technical cooperation on drugs and crime in the islamic republic of iran 20112014 [Internet]. 2014 [cited 2018 Apr 13]. Available from: http://www.unodc.org/islamicrepublicofiran

10. Islamic Republic of Iran | UNAIDS [Internet]. [cited 2018 May 30]. Available from: http:/www.unaids.org/en/regionscountries/countries/ islamicrepublicofiran

11. Islamic Republic of Iran AIDS Progress Report On Monitoring of the United Nations General Assembly Special Session on HIV and AIDS. 2015 [cited 2018 May 30]; Available from: http://www.unaids.org/ sites/default/files/country/documents/IRN narrative report 2015.pdf

12. Nikfarjam A, Hajimaghsoudi S, Rastegari A, Haghdoost $\bar{A} A$, Nasehi AA, Memaryan N, et al. The Frequency of Alcohol Use in Iranian Urban Population: The Results of a National Network Scale Up Survey [Internet]. Vol. 6, Int J Health Policy Manag. 2017. p. 97-102. Available from: http://www.ijhpm.com/article 3257.html

13. Maghsoudi A, Baneshi MR, Neydavoodi M, Haghdoost A. Network scale-up correction factors for population size estimation of people who inject drugs and female sex workers in Iran. PLoS One. 2014;9(11)

14. Jafari Khounigh A, Haghdoost AA, Salari Lak Sh, Zeinalzadehd AH, Yousefi Farkhade R, Mohammadzadehe M, et al. Size Estimation of Most-at-Risk Groups of HIV/AIDS Using Network Scale-up in Tabriz, Iran. J Clin Res Gov [Internet]. 2014;3(1):21-6. Available from: http://index.sciencepub.se/ojs/index.php/JCRG/article/view/80

15. Shokoohi M, Baneshi MR, Haghdoost AA. Size estimation of groups at high risk of HIV/AIDS using network scale up in Kerman, Iran. Int J Prev Med. 2012;3(7):471-6.

16. Sharifi H, Karamouzian M, Baneshi MR, Shokoohi M, Haghdoost AA, McFarland W, et al. Population size estimation of female sex workers in Iran: Synthesis of methods and results. PLoS One [Internet]. 2017 [cited 2018 Jan 18];12(8). Available from: http://journals.plos.org/

plosone/article/file?id=10.1371/journal.pone.0182755\&type=printable

17. Lansky A, Finlayson T, Johnson C, Holtzman D, Wejnert C, Mitsch A, et al. Estimating the number of persons who inject drugs in the United States by meta-analysis to calculate national rates of HIV and hepatitis C virus infections. PLoS One. 2014;9(5).

18. Sheikhzadeh K, Baneshi MR, Afshari M, Haghdoost AA. Comparing direct, network scale-up, and proxy respondent methods in estimating risky behaviors among collegians. J Subst Use [Internet]. 2016 [cited 2017 Oct 12];21(1):9-13. Available from: http://kmu.ac.ir/Images/ Archive/Comparing direct, network scale-up, and proxy respondent 4.pdf

19. Maghsoudi A, Jalali M, Neydavoodi M, Rastad H, Hatami I, Dehghan A. Estimating the prevalence of high-risk behaviors using network scale-up method in university students of Larestan in 2014. J Subst Use. 2017;22(2):145-8.

20. UNAIDS. 90-90-90 An ambitious treatment target to help end the AIDS epidemic. Http://WwwUnaidsOrg/Sites/Default/Files/Media Asset/90-90-90 En 0Pdf. 2014;40. 